in this relationship. With CS rates rising by $4 \%$ globally per year, we aimed to examine the relationship between mode of delivery and overweight/obesity in childhood.

Methods SLOPE is a linked population-based cohort of anonymised routine antenatal, birth and child healthcare records in Hampshire, UK (2003-2018). Delivery method was categorised into unassisted vaginal delivery, assisted vaginal delivery and CS (including elective and emergency). Child body mass index (BMI) was measured as part of the National Child Measurement Programme in England. Children were identified as overweight/obese if their age- and sex-adjusted BMI was above the 85 th percentile. Generalised linear modelling for outcome at two time points; 4-5 years $(n=30,229)$ and $10-11$ years $(n=14,305)$ was conducted, adjusting for clustering within families. Modelling was introduced in stages with the choice of covariates informed by a Directed Acyclic Graph, first adjusting for maternal BMI, then adding in confounders including maternal age, ethnicity, educational attainment, parity, smoking status at booking appointment, pre-eclampsia, and previous CS (model C) and then birthweight and gestational age at birth as potential mediators (model M). Analyses were also stratified by maternal BMI category (underweight: <18.5, normal weight: 18.5 to $<25$, overweight: 25 to $<30$, obese: $\geq 30$ $\mathrm{kg} / \mathrm{m}^{2}$ ) at booking.

Results Of children delivered by CS, $25.0 \%$ and 33.7\% were overweight/obese by $4-5$ years and $10-11$ years respectively, compared to $21.9 \%$ and $31.0 \%$ respectively with vaginal births. In unadjusted analysis, CS was associated with increased risk of overweight/obesity at 4-5 years (relative risk (RR) 1.13, 95\% Confidence Interval (95\% CI) 1.08-1.19), and at 10-11 years (RR 1.08, 95\% CI 1.02-1.14), however both were attenuated by adjusting for maternal BMI. In stratified analyses, CS delivery was associated with increased risk of childhood overweight/obesity at 4-5 years only in normal weight women (model C: RR 1.15, 95\% CI 1.04-1.27, model M: RR 1.14, 95\% CI 1.02-1.26), but not in 10-11 year models.

Conclusion Maternal weight status at the start of pregnancy is a strong confounder in the relationship between mode of delivery and childhood overweight/obesity. In stratified analyses, this association was evident only for children of normal weight women. If this relationship is causal, the potential mechanisms need to be explored.

\section{OP42 MATERNAL CHARACTERISTICS ASSOCIATED WITH VAPING IN PREGNANCY}

C Opondo*, S Harrison, F Alderdice, C Carson, MA Quigley. Nuffield Department of Population Health, University of Oxford, Oxford, UK

10.1136/jech-2020-SSMabstracts.42

Background The potential for harm to developing foetuses by tobacco products is well established. Studies have focused on cigarette smoking as a source of exposure. The use of electronic cigarettes (vaping devices) has increased in recent years. The Office for National Statistics (ONS) reports vaping data among the general public, with the most recent estimates showing a $5 \%$ prevalence among all women. However, there is no data on prevalence of vaping among pregnant women in the UK. This study sought to estimate the prevalence of vaping during pregnancy and to explore the factors and outcomes associated with vaping in a population-based sample of women giving birth in England.

Methods The study was a cross-sectional population-based postal survey of maternal and infant health and care, the National Maternity Survey (NMS) 2018. A total of 16,000 women aged $16+$ years who were living in England and who had given birth in 2017 were invited to participate. They were identified at random from birth records by the ONS, and surveyed at 6 months post-partum. Demographic and clinical questions about their pregnancy, labour, birth, and postnatal period were sent to them on a questionnaire. We estimated the prevalence of vaping and patterns of cigarette smoking in this group of women, and conducted regression analysis to explore associations between maternal characteristics and vaping, and between vaping and birth outcomes. Survey data were weighted to reduce the potential for nonresponse bias.

Results The crude prevalence of vaping among pregnant women was $2.8 \%$. This varied according to the pattern of cigarette smoking in pregnancy: $0.3 \%$ in never-smokers; $3.3 \%$ in ex-smokers; $7.7 \%$ in women who quit smoking when they became pregnant (pregnancy-inspired quitters); $9.5 \%$ in women who temporarily stopped smoking while pregnant but resumed smoking after the birth of their baby (temporary quitters); and $17.7 \%$ in women who continued to smoke during and after their pregnancy (persistent smokers). Younger women, unmarried women, women with fewer years of formal education, women living with a smoker, and persistent smokers (compared to quitters, ex- and never-smokers) were more likely to vape. Vaping was also associated with 3.2 weeks' reduction in the duration of breastfeeding and $10 \%$ reduction in the prevalence of breastfeeding for at least 8 weeks. However, there was no evidence of association between vaping and any of the maternal characteristics or birth outcomes after adjusting for the pattern of cigarette smoking in pregnancy.

Conclusion The prevalence of vaping among pregnant women in the NMS 2018 survey was low. Characteristics associated with vaping were broadly similar to those associated with smoking. Adverse birth outcomes and reduced breastfeeding among pregnant women who vape may be explained by their relatively higher prevalence of cigarette smoking.

\section{Thursday 10 September}

\section{Hospital Admissions}

\section{OP43 HOW MUCH OF A DOUBLE JEOPARDY ARE PRETERM BIRTH AND SOCIAL DISADVANTAGE? A POPULATION- BASED STUDY OF HOSPITAL ADMISSIONS IN ONE MILLION CHILDREN IN ENGLAND}

${ }^{1} \mathrm{MA}$ Quigley ${ }^{*},{ }^{1} \mathrm{~V}$ Coathup, ${ }^{2} \mathrm{~A}$ Macfarlane, ${ }^{3} \mathrm{~S}$ Johnson, ${ }^{4} \mathrm{~S}$ Petrou, ${ }^{1} \mathrm{O}$ Rivero-Arias, ${ }^{1} \mathrm{C}$ Carson, ${ }^{3} \mathrm{E}$ Boyle, ${ }^{1} \mathrm{JJ}$ Kurinczuk. 'NPEU, Nuffield Department of Population Health, University of Oxford, Oxford, UK; ${ }^{2}$ Department of Health Sciences, City University, London, UK; ${ }^{3}$ Department of Health Sciences, University of Leicester, Leicester, UK; ${ }^{4}$ Nuffield Department of Primary Care Health Sciences, University of Oxford, Oxford, UK

\subsection{6/jech-2020-SSMabstracts.43}

Background Children who are born preterm and those who are socially disadvantaged tend to have increased morbidity in childhood. Few studies are large enough and have 
sufficient follow-up to explore the size of these separate effects across childhood. We explored whether birth before full term (gestation 39-41 weeks) and social disadvantage represent a double jeopardy by comparing the size of these separate effects on rates of hospital admission from birth to 10 years.

Methods We linked routine data from birth registration and hospital admissions for all live, singleton births in England in 2005-2006. Children $(n=1,018,136)$ were followed up from discharge from their birth admission to age 10 years, death or study end (31st March 2015). Negative binomial regression models were used to estimate adjusted rate ratios (aRR) with $95 \%$ confidence intervals $(\mathrm{CI})$ for the effect of gestational age $(<32,32-33,34-36,37-38,39-41,42$ weeks) and area deprivation (quintiles) at birth on hospital admissions rates, stratified by age $(<1,1-4,5-10$ years). Population attributable fractions (PAFs) were estimated using the aRR for gestational age and area deprivation. All analysis was conducted in Stata version 14.

Results Hospital admission rates increased strongly with prematurity and area deprivation, even after adjustment for other factors. The effect of gestation was most marked in infancy, when even being born at 'early term' (gestation 3738 weeks) had a stronger effect than living in the most deprived areas $(\mathrm{aRR}=1.37$, 95\% CI $1.35-1.39$ for $37-38$ versus 39-41 weeks; $a R R=1.26$, 95\% CI 1.24-1.28 for the most versus least deprived areas). The effect of gestation persisted though was less marked at age 5-10 years, although even being born 'late preterm' (gestation 34-36 weeks) had a stronger effect than living in the most deprived areas $(\mathrm{aRR}=1.39,95 \%$ CI $1.35-1.43$ for 34-36 versus 39-41 weeks; $a R R=1.29$, 95\% CI 1.26-1.31 for the most versus least deprived areas). In infancy, the PAF for birth before full term $(<39$ weeks) was larger than the PAF for living in the poorest quintile. At age 5-10 years, the PAF for birth before full term was only slightly smaller than the PAF for living in the poorest quintile

Conclusion Gestational age and social deprivation were independent risk factors for hospital admissions throughout childhood. The impact of being born even a few weeks early is of similar magnitude to living in the most deprived areas. Children born early and living in more deprived areas have a double jeopardy.

\section{OP44 BORN TOO SOON: EVIDENCE FROM ONE MILLION CHILDREN ON HOW PREMATURITY AFFECTS HOSPITAL ADMISSIONS IN CHILDHOOD}

${ }^{1} V$ Coathup*, ${ }^{2} E$ Boyle, ${ }^{1} \mathrm{C}$ Carson, ${ }^{2} \mathrm{~S}$ Johnson, ${ }^{1} \mathrm{~J}$ Kurinzcuk, ${ }^{3} \mathrm{~A}$ Macfarlane, ${ }^{4} \mathrm{~S}$ Petrou, ${ }^{1} \mathrm{O}$ Rivero-Arias, ${ }^{1} \mathrm{M}$ Quigley. ${ }^{1}$ Nuffield Department of Population Health, University of Oxford, Oxford, UK; ${ }^{2}$ Department of Health Sciences, University of Leicester, Leicester, UK; ${ }^{3}$ School of Health Sciences, Division of Midwifery and Radiography, City University, London, UK; ${ }^{4}$ Nuffield Department of Primary Care Health Sciences, University of Oxford, Oxford, UK

\subsection{6/jech-2020-SSMabstracts.44}

Background Preterm children are at increased risk of morbidity throughout childhood. However, few studies have investigated the long-term health consequences in relation to the full spectrum of gestational age, week-by-week. This study aimed to estimate the effect of gestational age on hospital admissions from birth up to 10 years of age, explore how rates of hospitalisation change throughout childhood.
Methods Birth registration and hospital admission records were linked for all live, singleton births occurring in England between in 2005 and 2006. Children $(n=1,018,136)$ were followed up from birth admission hospital discharge to age 10, death or study end (31st March 2015). Generalised estimating equations were used to estimate rate ratios (RR) with 95\% confidence intervals (CI). The analysis was repeated looking at infection-related hospital admissions only.

Results Compared to children born at 40 weeks, those born $<28$ weeks had the highest rates of hospital admission throughout childhood $(\mathrm{aRR}=4.89,95 \% \mathrm{CI}=4.55-5.26)$. Even children born at 38 weeks had a higher rate of hospital admission during childhood compared to those born at 40 weeks $(\mathrm{aRR}=1.19,95 \% \mathrm{CI}=1.16-1.22)$. However, the effect of gestational age on hospital admission decreased as the children became older (interaction $\mathrm{p}<0.0001$ ). The most common cause of hospital admissions throughout childhood was infection and similar results were observed when the analysis was restricted to infection-related hospital admissions only e.g. $<28$ vs. 40 weeks, $\mathrm{aRR}=5.57,95 \% \mathrm{CI}=5.01-5.91$. Gestational age had the strongest effect on rates of lower respiratory tract infections, invasive bacterial and viral infection-related admissions.

Conclusion Gestational age is a strong predictor of childhood morbidity, particularly infection-based morbidity, with those born the most preterm at the highest risk. Whilst the effect of gestational age on hospital admission rates decreases with age, an excess risk remained at age 7-10 years.

\section{OP45 WHY DO PEOPLE ATTEND A\&E? A CROSS-SECTIONAL STUDY OF EMERGENCY DEPARTMENTS IN GREATER GLASGOW AND CLYDE}

KA Levin*, S Fleming, E Crighton. Public Health Directorate, NHS Greater Glasgow and Clyde, Glasgow, UK

\subsection{6/jech-2020-SSMabstracts.45}

Background There has been a year-on-year rise in attendance at A\&E departments (EDs) in Greater Glasgow and Clyde (GGC) since 2015/16, despite the opening of alternative units such as medical assessment units and minor injury units (MIUs), and subsequent falling emergency admission rates. This study aims to understand why people choose to attend EDs.

Methods All five EDs in GGC were surveyed over 3 weeks in November/December of 2019. Only those not in crisis were included in the survey. A total of 1196 people were surveyed. Qualitative methods were used to analyse the text data, while chi squared tests compared quantitative data between hospitals. Demographic representativeness was assessed using November/ December 2018 attendance data.

Results Less than a third of survey respondents believed that they had a medical emergency. $42 \%$ were advised to attend by a health practitioner. Twelve per cent attended at A\&E because they could not get a GP appointment with no significant difference between hospitals. Convenience was also cited as a factor and the feeling that this would be the quickest and best place for the person to attend. One third of the four ED respondents tried to get a GP appointment before coming to A\&E, ranging between $28.7 \%$ and $42.2 \%$ 